\title{
Research and Application of Communication between Siemens TDC and PLC based on DP
}

\author{
Ren Xiao-huai, Zhang Fei, Li Jing \\ Engineering Research Institute, University of Science and Technology Beijing, Beijing, China
}

\begin{abstract}
PLC always need to share in-out data each other on production control process. DP Net is widely applied at the advantage of simple structure, high and stable transmission speed. This article take the testing warm rolling mill for an example, introduce the PROFIBUS-DP communication between SIMATIC TDC and PLC-300. Through DP Net to realize the data switching, which TDC controller acts as DP master and PLC-300 acts as DP slave. The result of testing warm rolling mill's application proves the fact that DP Net can realize the communications between PLC at a high and stable speed.
\end{abstract}

Keywords-SIMATIC TDC, S7-300 PLC, PROFIBUS-DP Communication

西门子 TDC 与 PLC 的 DP 主从通讯研究及应用

任晓怀 张飞 李静

北京科技大学冶金工程研究院，北京，中国

摘 要 在生产控制中, 多个 PLC 之间经常需要共享输入输出的数据。DP 网以结构简单, 传输速度高且稳定而被广泛应用。本 文以温轧实验轧机为例, 介绍 SIMATIC TDC 与 PLC-300 之间的 PROFIBUS-DP 通讯, 通过 DP 网, 实现以 TDC 控制器作为 DP 主站, PLC-300 控制器作为 DP 从站的数据交换。现场实验温轧机的应用情况也很好的证明了 DP 网能高速稳定的实现 PLC 之间的通讯。

关键词 SIMATIC TDC, S7-300 PLC, PROFIBUS-DP 通讯

\section{1. 引言}

西门子公司是欧洲最大的 PLC 生产厂家之一, 其 S7 系列 PLC 提供了强大的控制功能、网络通讯和 IT 服务功 能, 并且可以方便的进行系统维护、热拔插和远程诊断等。 西门子计算机系统是现在采用比较多的工厂自动化过程控 制系统, 其具有以下特点: 强大的计算和工艺功能; 超大 的内存容量, 可以集成新的功能; 尺寸小, 大量集成的功 能; 高效的工程软件, 统一的编程组态; 分布式自动化系 统, 减少安装和调试的时间、费用; 高可靠性和强大的诊 断功能。基于上述特点, 许多工业现场都采用了西门子公 司的产品。

在工业现场, 控制和工艺要求复杂, 所以控制器与控 制器之间要经常互相通讯, 共享数据。常用通讯方式有:
Industrial Ethernet、MPI、PROFIBUS、TCP/IP 等, 其中 PROFIBUS-DP 现场总线通讯结构精简、传输速度快、实时 性好并且易于扩展 ${ }^{[1]}$, 很多工业现场都采用了 DP 网通讯方 式。

\section{2. 设备及网络结构}

实验温轧机是在实验室原冷轧机基础上进行改造而 成, 主要由放料架、放卷机、前张力辊、前加热炉、轧机 本体、后加热炉、后张力辊、收卷机、液压系统及电气自 动化设备等组成 (见图 1)。电气自动化部分主要包括一台 SIMATIC TDC, 一台 ET200M 站、一台 PLC-300 站以及两 台西门子 6SE70 系列的变频器。

中央高校基本科研业务费专项资金资助 


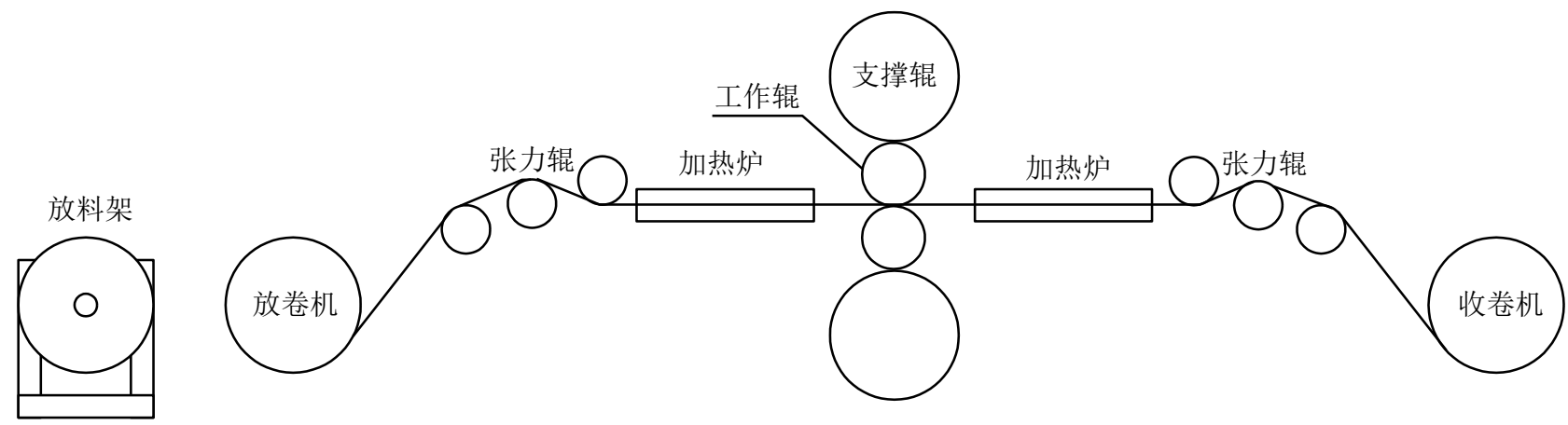

图 1 温轧机设备结构图

\subsection{SIMATIC TDC}

SIMATIC TDC ( SIMATIC Technology and Drive Control）控制器是西门子公司推出的一种适用于多处理器 的自动化系统, 擅长解决处理复杂的驱动、控制和通讯任 务。其突出的特性有: 模块化结构, 可扩展; 采样时间可 达 $100 \mathrm{~ms}$, 循环时间达 $0.1 \mathrm{~ms}$; 采用 64 位结构（RISC）的 中央处理器; 同步多处理器运行, 每个机架可有 20 个 CPU; 可耦合 44 个机架; 编程采用连续功能图 (CFC) 和顺序功 能图 (SFC) [2]。

SIMATIC TDC 系统还提供数字量和模拟量 I/O 模板、 工业以太网模板、PROFIBUS-DP/MPI 模板、增量型和绝对 值型高速计数器模板等 ${ }^{[3]}$, 扩展了 SIMATIC TDC 性能。多 处理器运行方式实现了性能的几乎无限制扩展。SIMATIC $\mathrm{TDC}$ 是一种可靠的, 面向未来的系统, 在提高生产质量的 同时也提高了生产效率。

实验温轧机采用 CPU551 模板和 CP50M1 通讯模板 (见 图 2)。

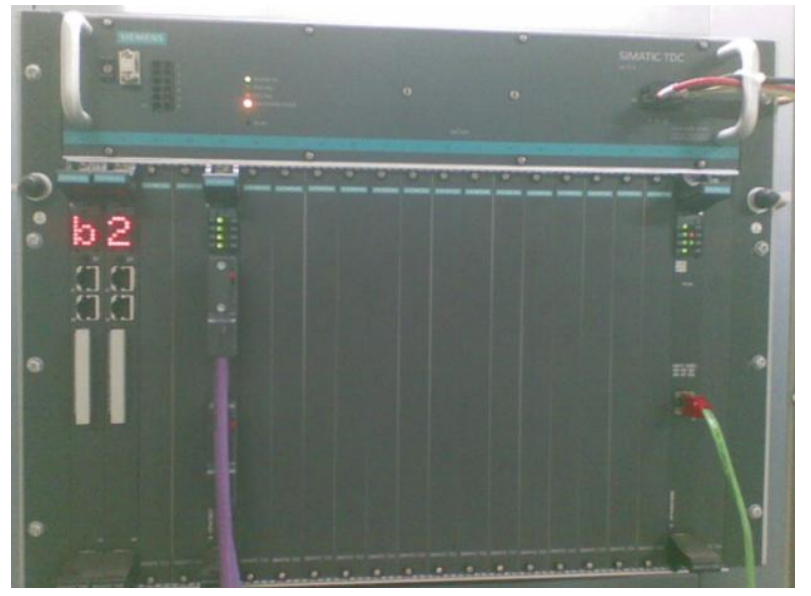

图 2 SIMATIC TDC 控制器

\subsection{ET200M 和 PLC-300}

ET200M 是高密度配置的模块化 $\mathrm{I} / \mathrm{O}$ 站, 可以作为 S7-300 或 S7-400 等控制器的信号、功能和通讯模块扩展, 主要由以下部分组成: IM153 接口、各种 I/O 模块、电源 (可无)。

PLC-300 是西门子推出的中低端可编程逻辑控制器, 主要由中央处理单元 $(\mathrm{CPU})$ 、电源单元 $(P S)$ 、信号模板 $(\mathrm{SM})$ 、接口模板 (IM)、功能模板 (FM)、通讯模板 (CP) 等组成。

\subsection{PROFIBUS-DP 网}

ProfiBus 是在欧洲工业界得到最广泛应用的一个现场 总线标准, 也是目前国际上通用的现场总线标准之一。它 属于单元级、现场级的 SIMATIC 网络, 适用于中、小量的 数据传输。ProfiBus 区分主站和从站: 主站掌握总线中数 据的控制权, 从站不拥有总线访问的授权, 只是简单的输 入输出设备。只要符合 PROFIBUS-DP 规约的设备都可以 加入到 PROFIBUS 网作为主站和从站。ProfiBus 现场应用 提供了三种通信协议: PROFIBUS-FMS、PROFIBUS-DP、 PROFIBUS-PA。其中 PROFIBUS-DP 用于与分布式的 I/O 或者一些智能从站进行高速通讯, 通过一种快速的串行接 口使控制器与输入输出设备通信。PROFIBUS-DP 是一种廉 价的传输方式, 传输介质采用双绞线, 传输速度为 $9.6 \mathrm{Kbps}$ $-12 \mathrm{Mbps}$ 。

温轧机自动控制系统的整个网络结构由 TCP/IP 和 DP 两种通信方式构成。ET200M 和 PLC-300 作为 DP 从站通 过 DP 网与 SIMATIC TDC 主站通讯, TDC 通过 TCP/IP 与 HMI 通讯, 两台变频器通过 DP 网与 TDC 控制器连接。网 络结构如图 3 所示。 


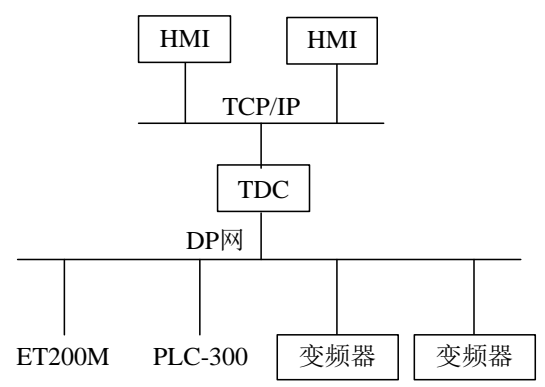

图 3 网络结构

\section{3. 系统构建}

\section{1 组态 PLC-300 从站}

打开 PLC-300 硬件组态, 按照现场设备的配置, 对 S7-300 站进行组态, 实验温轧机 PLC-300 从站组态结果如 图 4 所示。

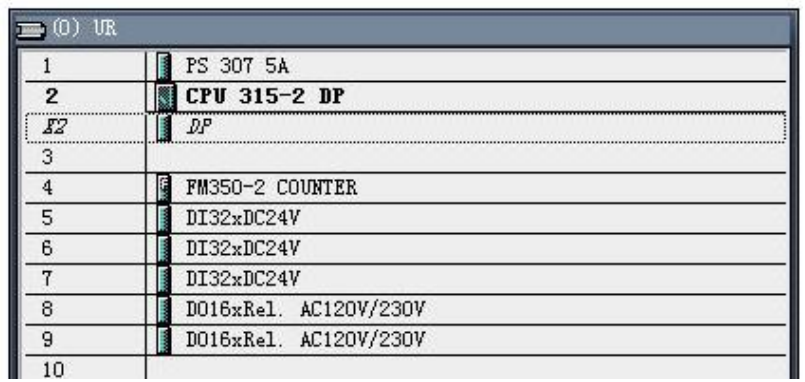

图 4 PLC-300 硬件组态

硬件组态完成以后, 要配置 PLC-300 站的 DP 网参数: DP 网地址设为 4; “Operating Mode” 选择为 DP slave; “Configuration” 栏中创建一个输入域和一个输出域, 按照 实际需要选择域长度, 长度单位为字节 (Byte), 最大为 32 个字节。此例中长度分别设为 6 字节和 20 字节; “Unit” 中选择按字节方式来通信; “Consistency” 中选择 “Unit”, 表示按照 “Unit” 中定义的方式来进行通信。完成以后, 编译并保存硬件组态, 就完成了对 PLC-300 智能从站的硬 件组态。

\section{2 组态 SIMATIC TDC 主站}

打开 TDC 硬件组态, 对 TDC 主站进行组态。本例中 1,2 插槽为 CPU551 模板, 第 5 插槽为 CP50M1 通讯模板。 在 CP50M1 模板 DP 接口新建一个 PROFBUS-DP 网: 地址 为 2; Operating Mode 选为 DP master; 在新建的 PROFIBUS 网上插入两个从站: ET200M 和 PLC-300。方法为: 在右 侧目录选中 PROFIBUS DP-Configured Stations-CPU31X 和 PROFIBUS DP-ET200M-IM153-1, 分别添加到新建 的 DP 网上（见图 5)，完成通讯网络的组态。

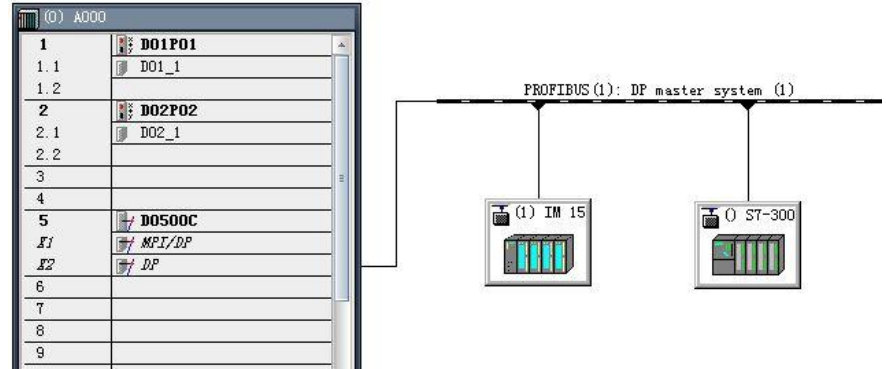

图 5 SIMATIC TDC 硬件组态

DP 网组态好以后, 需进行主站和从站的连接。完成以 后, 在 “Configuration” 配置栏中编辑输入输出域, 使 DP Master 的输入域对应 S7-300 站的输出域, 输出域对应输入 域 ${ }^{[4]}$ 。选中 ET200M 从站, 完成对它的组态（见图 6)。编 译并保存，完成对 TDC 主站的硬件组态。

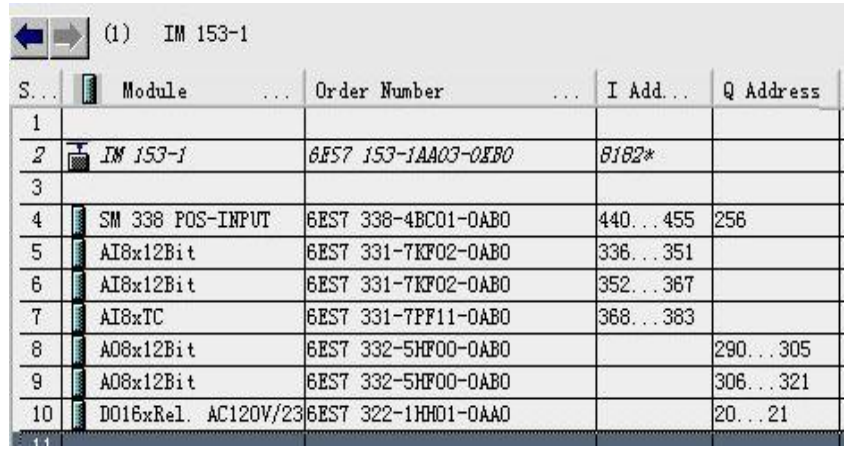

图 $6 \mathrm{ET} 200 \mathrm{M}$ 硬件组态

\section{3 编程}

硬件组态完成以后, 需要编写通讯程序来实现 DP 主 站和从站之间的通讯。通讯块主要采用 CRV_P 和 CTV_P 分别读取和发送数据, 图 7 是本例中用于接收和发送数据 的通讯块。

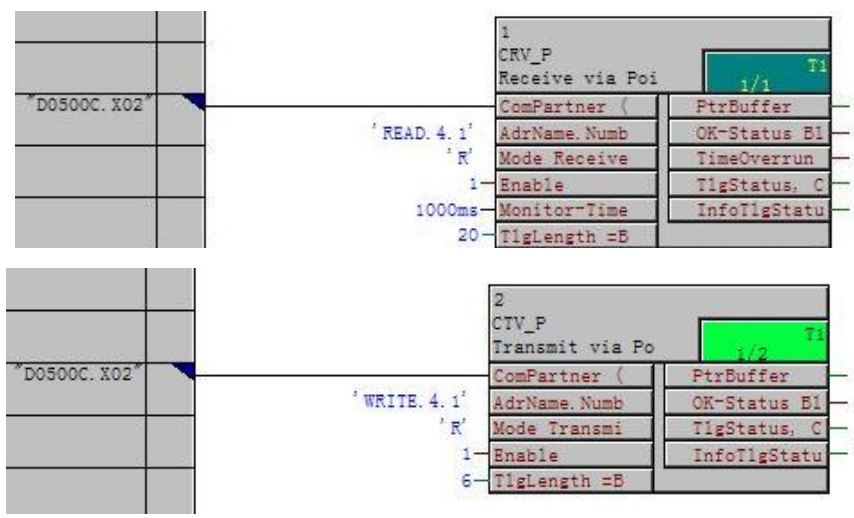

图 7 CRV_P, CTV_P 功能块 
两个模块的各个参数含义[5]如下:

CTS-ComPartner(接口、模块), 硬件通讯模块的接口， 此例连接的是 CP50M1 模块的 X02 DP 接口。

AR一AdrName.Number, 通道编号, 该编号在发送器 和接收器之间建立所需连接, 格式为: 通道名. 从站地址. 数 据格式, 如 “READ.4.1” 和 “WRITE.4.1”。

MOD-Mode, 接收模式, “ $R$ ” 为刷新, “ $H$ ” 为同步 交换。

$\mathrm{EN}$-enable, 启用, 当 $\mathrm{EN}=0$ 时, 不处理块。

TMX-Monitor-Time, 监视时间, 即需要在指定的时 间内接收报文，否则报错。

NBY一TlgLength, 缓冲区大小, 以字节为单位。

CRV_P 和 CTV_P 是基于指针的接收发送块, 只要定 义好指针接口, 就可以读写指针所指的数据区的数据。在 TDC 中编写通讯程序之后, PLC-300 侧无需编写任何通讯 程序, 就可完成 TDC 与 PLC-300 的 DP 通信。

\section{4. 结束语}

基于 DP 网结构简单、传输速度高且稳定的优点, 实 验温车机采用 DP 网组建通讯网络, 并实现 TDC 控制器与
ET200M 从站、PLC-300 从站和变频器的数据共享。从现 场的应用效果可以看出, DP 网的组建和应用非常简单, 传 输速度稳定且快速, 实时性非常好, 满足了现场控制的需 求。

\section{参考文献(References)}

[1] Huang Zheng, Gu Peng. Research and design of communication system based on S7 PLC. Electrical engineering technology, 2010, 39(06): 45 48.

[2] Siemens (China) Ltd, SIMATIC TDC User manual. 2006.

[3] Zhang Fei, Yang Quan, Hu Shui-ping. Automatic control system of experimental mill in tandem cold rolling. Experimental Technology and Management, 2011, 28(2): 73 74.

[4] Siemens (China) Ltd, The ProfiBus communication between PLC and PLC. 2004.

[5] Siemens (China) Ltd, SIMATIC TDC Function block manual, 2004. 